\title{
Single-application Radiofrequency Interruption in a Broad Isthmus Ventricular Tachycardia by Targeting the Longest Electrogram Visualized Using a New Customized Software (VEDUMap)
}

\author{
FILIPPO M. CAUTI, MD, ${ }^{1}$ STEFANO BIANCHI, MD, ${ }^{1}$ and PIETRO ROSSI, MD, PhD ${ }^{1}$ \\ ${ }^{1}$ Arrhythmology Unit, Ospedale San Giovanni Calibita, Fatebenefratelli Hospital, Isola Tiberina, Rome, Italy
}

KEYWORDS. Slow conduction, VEDUMap, ventricular tachycardia ablation.

ISSN 2156-3977 (print)

ISSN 2156-3993 (online)

CC BY 4.0 license

(C) 2021 Innovations in Cardiac

Rhythm Management
A 63-year-old woman with a diagnosis of arrhythmogenic right ventricular cardiomyopathy was referred to our department for recurrent monomorphic ventricular tachycardia (VT) with left bundle branch block and intermediate axis. A previous endocardial procedure was ineffective. After pericardial access, a detailed electroanatomical sinus rhythm map was obtained to verify areas of slow conduction and local abnormal ventricular activation (Figure 1B). A confined spot of late potentials (LPs) was detected in an inferolateral aneurism. A marked deceleration zone in sinus rhythm was revealed at the base of the apex of the spot of LPs. VT was induced with a single extrastimulus and the full cycle length was recorded in the epicardium by the Advisor ${ }^{\mathrm{TM}}$ HD Grid Mapping Catheter, Sensor Enabled ${ }^{\mathrm{TM}}$ using the HD Wave acquisition algorithm (Figure 1A). The VT isthmus revealed a

The authors report no conflicts of interest for the published content. Address correspondence to: Filippo Maria Cauti, MD.

Email: filippocauti@hotmail.it. broad path with inferior entrance and anterolateral exit in the epicardial base of the right ventricle. Thus, a novel ventricular map (VEDUMap) of electrogram (EGM) duration (unpublished data), which considered the duration of the EGM in a color-coded fashion (white = longest to purple $=$ shortest) displayed with auto-color was created (Figure 1C). The region of the prolonged EGM during VT corresponds to the slowest conduction in sinus rhythm. Radiofrequency $\left(50 \mathrm{~W}\right.$ at $43^{\circ} \mathrm{C}$ with an open irrigated catheter) (FlexAbility ${ }^{\mathrm{TM}}$ ablation catheter) was started at the white spot highlighted by the VEDUMap with sudden interruption of the tachycardia (green dot in Figure 1) (Video 1).

After a single RF pulse, the VT was no longer inducible up to the fourth extrastimulus. A line of radiofrequency energy was delivered between the two VT isthmus boundaries. LPs were abolished and confirmed by remapping. The patient remained free from arrhythmia at 11 months of follow-up. 


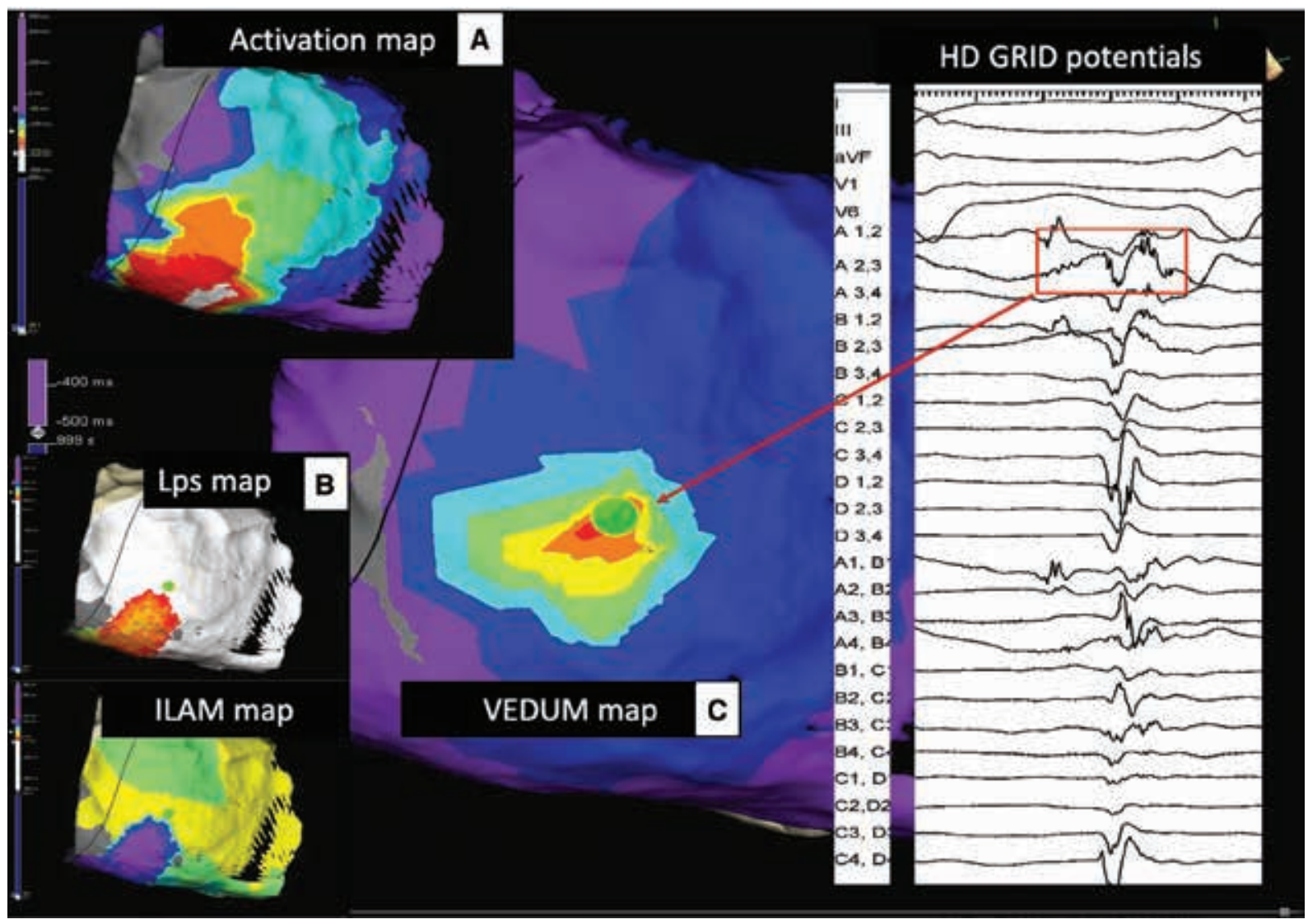

Figure 1: A: Activation map with first deflection detection shows a broad VT isthmus with an entrance on the inferior epicardial wall (white) and exit towards the right ventricular epicardial base. B: Sinus rhythm maps (local activation time map) with LPs map and isochronal late activation map. C: Ventricular map of EGM duration shows the crucial spot of VT interruption. The EGM recorded by the A2,3 bipoles (white color in the VEDUMap) in the EGM cover more than $60 \%$ of the diastolic phase. The green dot indicates the site of radiofrequency interruption. 